\title{
Deep Three-Dimensional Solid-State Qubit Arrays with Long-Lived Spin Coherence
}

\author{
C. J. Stephen $\odot,{ }^{1}$ B. L. Green $\odot,{ }^{1}$ Y. N. D. Lekhai, ${ }^{1}$ L. Weng, ${ }^{2}$ P. Hillø $, 3,4$ S. Johnson, ${ }^{2}$ \\ A. C. Frangeskou, ${ }^{1}$ P. L. Diggle, ${ }^{1,4}$ Y.-C. Chen, ${ }^{2}$ M. J. Strain ${ }^{\circledR},{ }^{3,4}$ E. Gu, ${ }^{3,4}$ M. E. Newton, ${ }^{1,4}$ \\ J. M. Smith, ${ }^{2,4}$ P. S. Salter, ${ }^{5}$ and G. W. Morley ${ }^{1,4, *}$ \\ ${ }^{1}$ Department of Physics, University of Warwick, Gibbet Hill Road, Coventry CV4 7AL, United Kingdom \\ ${ }^{2}$ Department of Materials, University of Oxford, Parks Road, Oxford OX1 3PH, United Kingdom \\ ${ }^{3}$ Institute of Photonics, University of Strathclyde, George Street, Glasgow G1 1RD, United Kingdom \\ ${ }^{4}$ EPSRC Centre for Doctoral Training in Diamond Science and Technology, University of Warwick, Coventry \\ CV4 7AL, United Kingdom \\ ${ }^{5}$ Department of Engineering Science, University of Oxford, Parks Road, Oxford OX1 3PJ, United Kingdom
}

(Received 25 May 2019; revised manuscript received 17 September 2019; published 3 December 2019)

\begin{abstract}
Nitrogen-vacancy centers (NVCs) in diamond show promise for quantum computing, communication, and sensing. However, the best current method for entangling two NVCs requires that each one is in a separate cryostat, which is not scalable. We show that single NVCs can be laser written $6-15-\mu \mathrm{m}$ deep inside of a diamond with spin coherence times that are an order of magnitude longer than previous laserwritten NVCs and at least as long as naturally occurring NVCs. This depth is suitable for integration with solid immersion lenses or optical cavities and we present depth-dependent $T_{2}$ measurements. 200000 of these NVCs would fit into one diamond.
\end{abstract}

DOI: 10.1103/PhysRevApplied.12.064005

\section{INTRODUCTION}

Demonstrated qubit fidelities for a single nitrogenvacancy center (NVC) and its nearby nuclear spins [1] are above the required thresholds for fault-tolerant quantum computing [2]. In addition, two NVCs in different cryostats have been optically entangled [3,4]. However, useful faulttolerant quantum computing will require $10^{6}-10^{9}$ physical qubits [2]. It will not be practical to build this with one cryostat per NVC so it is important to consider how many NVCs can be used inside of one diamond as inspired by previous experiments [1,5-7] and theoretical proposals [8-11]. We focus on optical entanglement rather than the possibility of having NVCs so close to each other that they directly interact magnetically [12] because no scalable fabrication technique has been found for the latter approach. For communications [4,13], having an array of NVCs will provide many spin-photon interfaces within one cryostat [14], increasing data rates and allowing multiplexing. Sensing with two-dimensional (2D) arrays of

\footnotetext{
*gavin.morley@warwick.ac.uk
}

Published by the American Physical Society under the terms of the Creative Commons Attribution 4.0 International license. Further distribution of this work must maintain attribution to the author(s) and the published article's title, journal citation, and DOI.
NVCs will combine the high resolution of single NVC sensing [15-17] with the simultaneous imaging achieved with wide-field microscopy [18]. Stacking two of these $2 \mathrm{D}$ arrays will then increase the sensitivity by subtracting the background noise measured by the array that is further from the sample of interest.

For all these technologies, we envision a fiber array or a spatial light modulator (SLM) that simultaneously sends and receives optical photons to and from a 2D array of NVCs through a lens or a microlens array. A solid immersion lens (SIL) array would be used to collect more of the fluorescence $[3,4,19,20]$ or an optical cavity array [21] would be used to improve the optical performance of NVCs in the middle of a diamond that is thin, for example, $4 \mu \mathrm{m}[22,23]$. For sensing, the NVCs need to be approximately $10 \mathrm{~nm}$ from the surface with no SIL or cavity, so delta doping with nitrogen would be used to control the depth of NVCs with a precision of $4 \mathrm{~nm}$ [24], followed by plasma etching to leave them at the surface.

The creation of 2D NVC arrays has been demonstrated previously [24-35], but not three-dimensional (3D) arrays. With ion implantation through a mask, high-precision placement of $10 \mathrm{~nm}$ [26] has been shown with electron spin $T_{2}$ times of up to $50 \mu \mathrm{s}$. Longer $T_{2}$ times of up to $530 \mu \mathrm{s}$ were achieved by using isotopically pure ${ }^{12} \mathrm{C}$ diamond, but with less precise placement and again requiring ion 
(a)

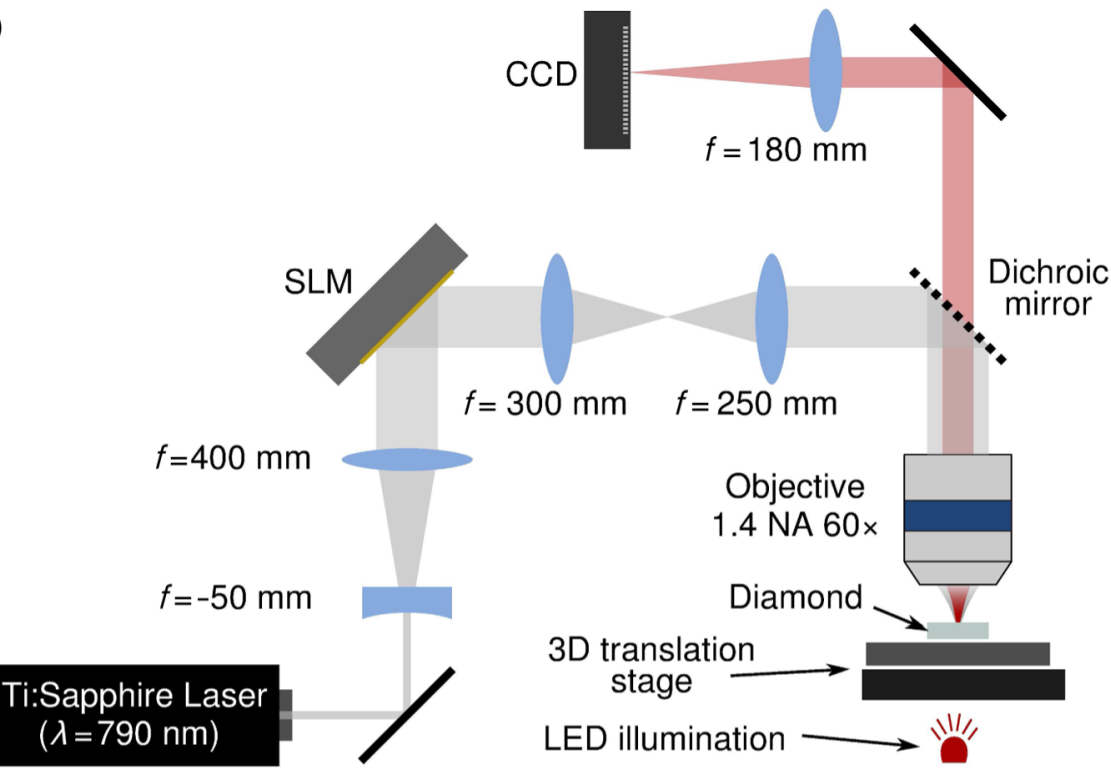

FIG. 1. Laser writing of 3D arrays of nitrogen vacancy centers in diamond with long spin coherence. (a) Schematic of the laserwriting setup. SLM is a spatial light modulator. CCD is a camera. (b) Wide-field transmission microscopy of the laser-written markers surrounding the arrays. The large boxes labeled A-G are $110 \mu \mathrm{m}$ across.

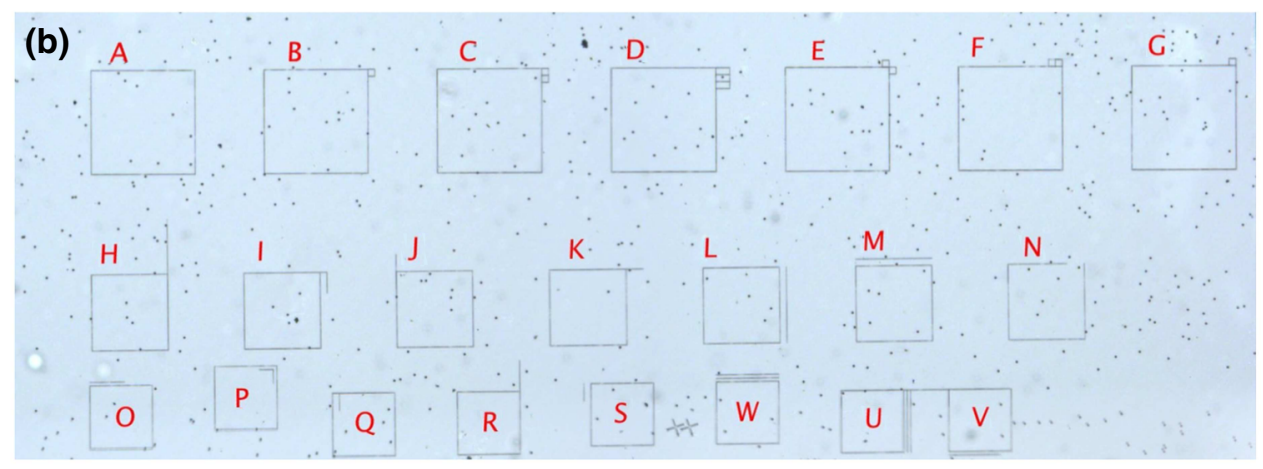

implantation through a mask [27]. For quantum computing, it is important to use diamond with natural isotopic abundance because the $1.1 \%{ }^{13} \mathrm{C}$ nuclear spins provide a valuable register of five or more qubits that can be used to store quantum information for longer than the electron spin [5-7,36]. Ion implantation has been shown to lead to cryogenic optical transitions that are too broad for quantum computing applications [37]. In contrast, laser writing similar to that presented here has produced transform-limited cryogenic optical transitions including photoluminescence excitation with linewidths below $14 \mathrm{MHz}$ [25]. Localized electron irradiation into a diamond provided a $2 \mathrm{D}$ array with a $T_{2}$ of up to $1.3 \mathrm{~ms}$ due to the use of ${ }^{12} \mathrm{C}$ diamond and no need for a mask [24]. Delta doping was used to reach a depth precision of $4 \mathrm{~nm}$ with an in-plane precision of $450 \mathrm{~nm}$. Laser-written optical waveguides have been made in diamond and optically detected magnetic resonance (ODMR) measured on the NVC ensembles inside [38,39]. 2D arrays of NVC ensembles have been created on the diamond surface with short-pulse laser nanoablation [40]. Earlier work from our collaboration has shown that 2D arrays of NVCs (some single, some double, some triple) can be laser written with no mask, but the $T_{2}$ time measured was typically only $30-80 \mu \mathrm{s}$ [25]. These short times may have been due to the creation of too much damage by overly energetic laser write pulses. Recent work from our collaboration describes preferential orientation and near $100 \%$ yield for $5 \times 52 \mathrm{D}$ arrays of laser-written NVCs in diamond, but the $T_{2}$ times are $170 \mu \mathrm{s}$ or less [41]. This reduced spin coherence may be due to the high concentration of nitrogen currently needed for the in situ annealing technique used or it may be that the in situ annealing does not heal the damage as well as traditional annealing in a furnace. The literature on 2D NVC arrays does not report the dependence of $T_{2}$ on NVC depth. The depth dependence of NVC $T_{2}$ times has been studied for shallow NVCs, finding that $T_{2}<100 \mu \mathrm{s}$ [42], which is good for nanoscale sensing but too short for quantum computing.

In this paper, we present the first 3D arrays of NVCs and find that their spin coherence times are consistently as long as naturally occurring NVCs over a range of depths. These coherence times are the result of (a) plasma etching to remove the subsurface polish damage, (b) a global thermal anneal, (c) high purity diamond, and (d) 
(a)

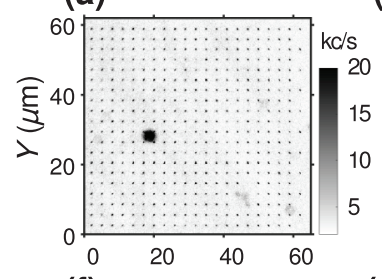

(b)

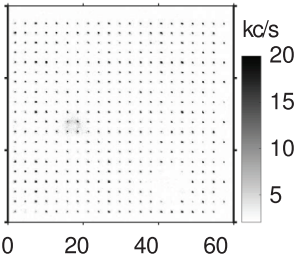

(c)

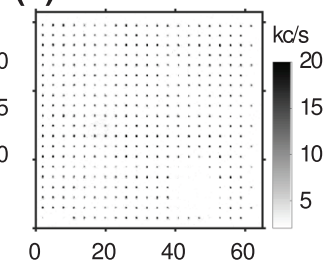

(d)

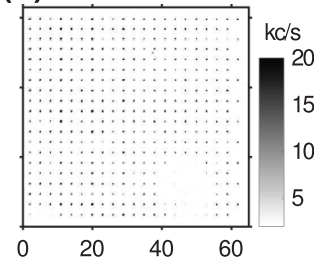

(e)

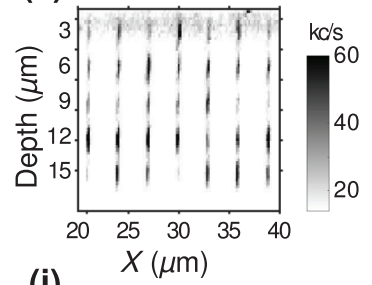

(j)
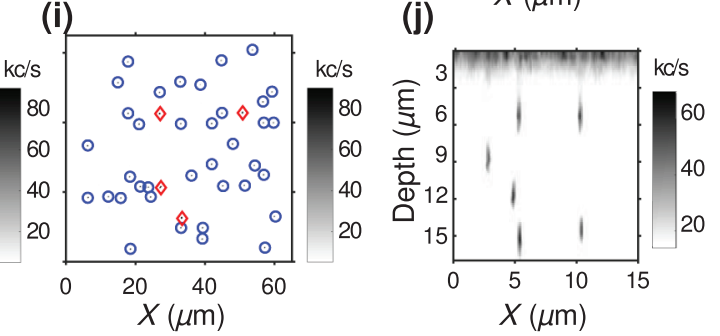

FIG. 2. Confocal imaging of a three-dimensional array of defects in diamond. Top row: (a)-(d) are in the $X$ - $Y$ plane at depths of 6,9, 12 , and $15 \mu \mathrm{m}$, respectively, before annealing: each spot is an ensemble of vacancies. (e) A vertical section in the $X$ - $Z$ plane. Bottom row: The same volume after annealing out the vacancies: the spots are nitrogen vacancy centers. In $9 \%$ of the target sites, NVCs are created, almost all as single centers (circled).

precisely calibrated laser-write-pulse energy to avoid creating unnecessary amounts of damage.

\section{FABRICATION WITH LASER WRITING}

Our 3D arrays are created by laser writing over 2000 NVCs into a diamond with a natural isotopic abundance of ${ }^{13} \mathrm{C}$. Figure $1(\mathrm{a})$ is a schematic of the writing process and Fig. 1(b) is an overview of all of the arrays written. Figure 2 shows one of the 3D arrays in the form of four
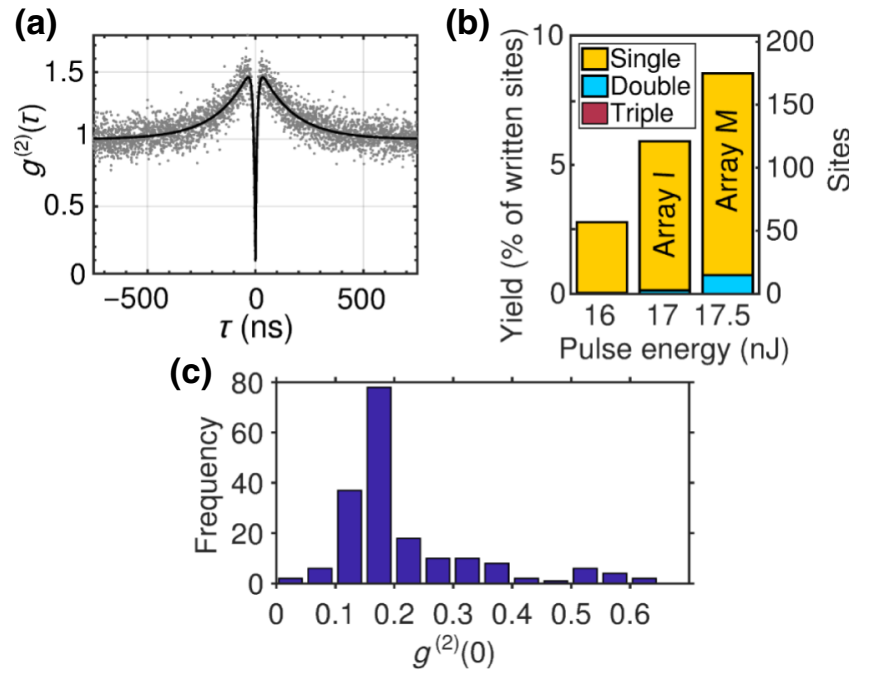

FIG. 3. Fraction of single nitrogen vacancy centers compared to doubles and triples. (a) HBT measurement of the photon arrival time for NVC1. (b) HBT measurements reveal the fraction of single, double, and triple NVCs produced in three of the $3-\mu \mathrm{m}$ pitch arrays with different laser-write pulse energies. (c) The frequency of the measured $g^{2}(0)$ for array $\mathrm{M}$ with no background subtraction.
2D images. We measure the spin-echo coherence time for 22 of the single centers and find that 15 of them have $T_{2}>500 \mu \mathrm{s}$ at room temperature. All our measurements are at room temperature where the electron spin coherence time is limited by the natural isotopic abundance of ${ }^{13} \mathrm{C}$. We use dynamic decoupling to probe the electron spin coherence without ${ }^{13} \mathrm{C}$ limitations, finding a coherence time limited by the electron spin $T_{1}$ as has been reported for naturally occurring NVCs.

To create the arrays, an electronic grade (EL grade) diamond is bought from Element Six, and plasma etching is used to remove $20 \mu \mathrm{m}$ of subsurface polishing damage $[17,43]$. Arrays of ensembles of vacancies are generated in the diamond lattice by single 250 -fs pulses from a 790 -nm laser focused tightly beneath the surface of the diamond using a high numerical aperture (NA) oil objective. The light-matter interaction is highly nonlinear, limiting any material modification to the center of the focal volume and giving an inherent three-dimensional resolution to the fabrication [44]. The refractive index mismatch at the oil-diamond interface causes refraction leading to a depth-dependent spherical aberration of the laser focus, which can limit three-dimensional fabrication resolution. Adaptive optics using a liquid crystal SLM is used to correct for the aberration [45], ensuring that the fabrication is the same at each depth. The full width at half maxima of the intensity distribution of the laser focus inside the diamond are theoretically estimated to be $350 \mathrm{~nm}$ radially and $1.7 \mu \mathrm{m}$ longitudinally. However, the expected dimensions over which the light-matter interaction is appreciable are likely to be much lower [46]. The diamond sample is mounted on a three-axis precision translation stage and moved relative to the laser focus to fabricate arrays of vacancy ensembles. 
(a)
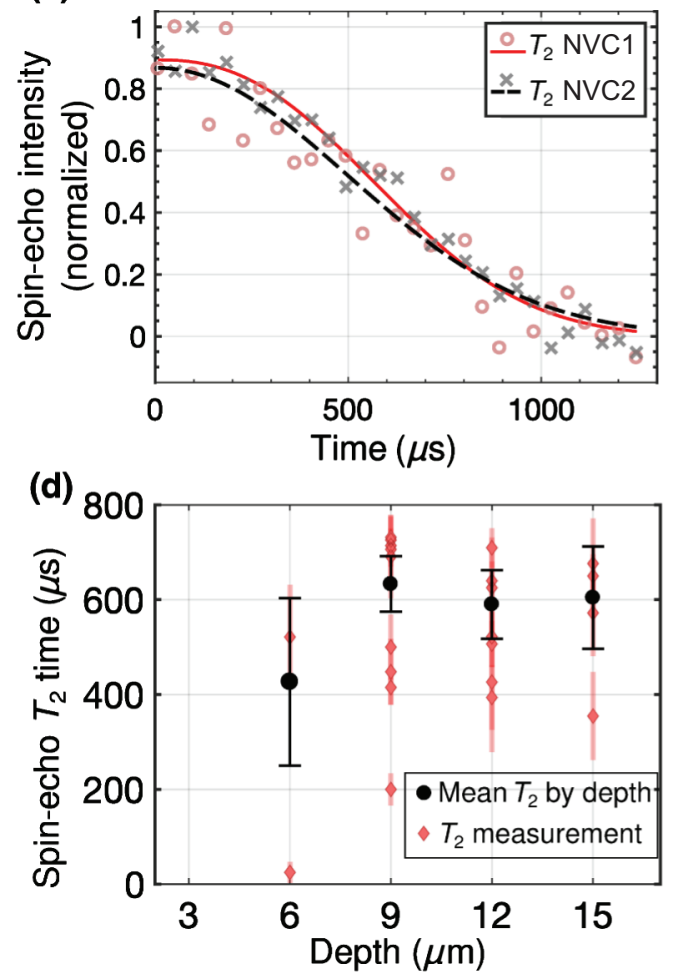

(b)

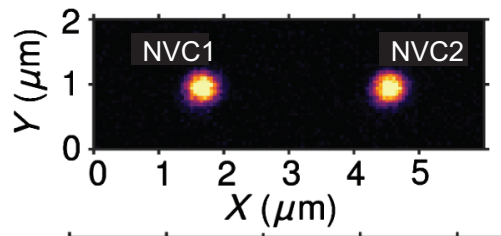

(c)

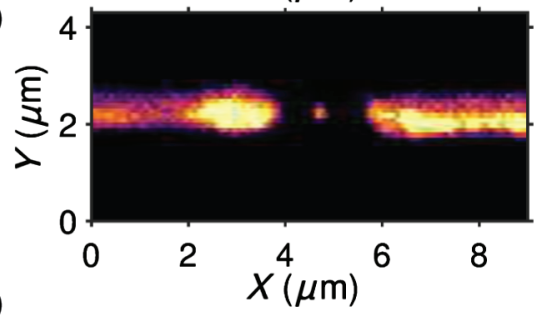

(e)

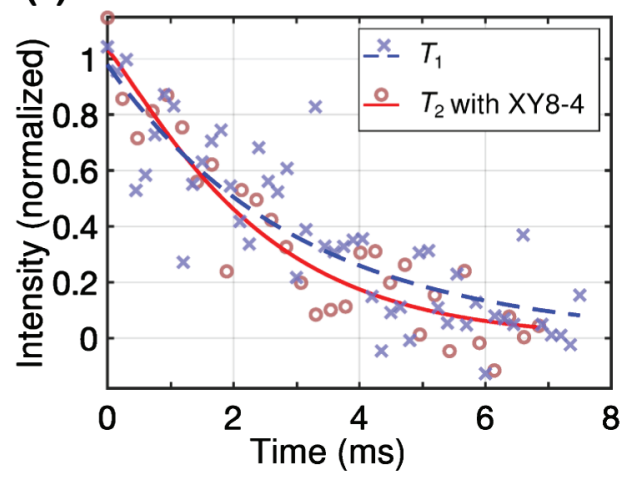

FIG. 4. Nitrogen vacancy center electron spin coherence times. (a) Spin-echo decays for adjacent, aligned NVCs [labeled NVC1 and NVC2, as shown in Figs. 4(b) and 1(g)] fitted with $A e^{-\left(t / T_{2}\right)^{n}}$. NVC1 has $T_{2}=710 \pm 40 \mu$ s with $n=2.4 \pm 0.4$, while NVC2 has $T_{2}=690 \pm 90 \mu$ s with $n=2.0 \pm 0.8$. (b) Enlarged confocal image of NVC1 and NVC2. (c) Confocal image of a NVC between two laser-written electrical wires from another region of the same diamond. (d) Spin-echo coherence times measured for sites in arrays $\mathrm{M}(17.5 \mathrm{~nJ})$ and $I(17 \mathrm{~nJ})$, which have a 3- $\mu \mathrm{m}$ pitch, as a function of NVC depth. (e) Using XY8-4 dynamic decoupling achieves $T_{2}=2.4 \pm 0.6 \mathrm{~ms}$ with $n=1.1 \pm 0.4 \mathrm{on} \mathrm{NVC1}$. For comparison, the longitudinal lifetime, $T_{1}$, of this site is $3.0 \pm 0.7 \mathrm{~ms}$.

In order to find the fine range of pulse energies to use in these experiments, an initial calibration study is carried out on the same fabrication run inside a nominally identical diamond by writing arrays of points across a coarse range of pulse energies. This sample is subsequently characterized using a scanning confocal microscope to find the pulse energy that produces just visible vacancy ensembles using an air objective. Previous work has shown that writepulse energies slightly lower than this are optimal for NVC creation [25]. Twenty-two 3D arrays labeled A to $\mathrm{V}$ are laser written with different energies from 14 to $19 \mathrm{~nJ}$ and with different pitches from 2 to $5 \mu \mathrm{m}$, with each 3D array having $21 \times 202 \mathrm{D}$ arrays stacked with up to six depths for a total of over 44000 writing sites. Once set up, laser writing of a 2000-point array typically takes $5 \mathrm{~min}$. Following laser writing and characterization, the sample is buried in diamond grit (from Element Six) and annealed for three hours at $1000^{\circ} \mathrm{C}$ in a tube furnace in a nitrogen atmosphere to convert the introduced vacancies into NVCs [25]. (a)

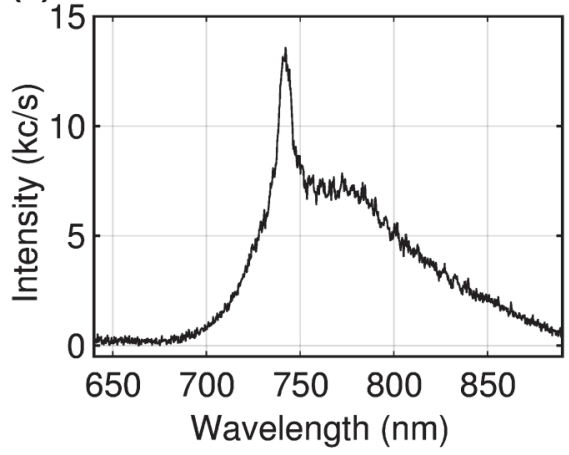

(b)

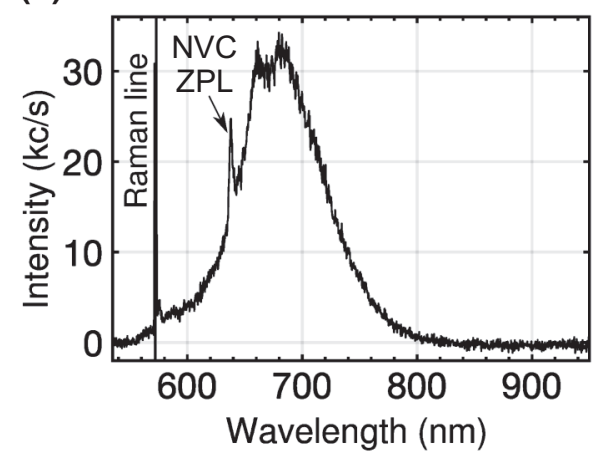

FIG. 5. Photoluminescence spectrum of (a) a single site at $25-\mu \mathrm{m}$ depth in array A $(19 \mathrm{~nJ})$ before the sample was annealed at $1000^{\circ} \mathrm{C}$. (b) A single site M9-4 (labeled NVC2 above), which shows the characteristic emission of $\mathrm{NVC}^{-}$(a negatively charged NVC) with a zero phonon line (ZPL) at $637.7 \mathrm{~nm}$. 


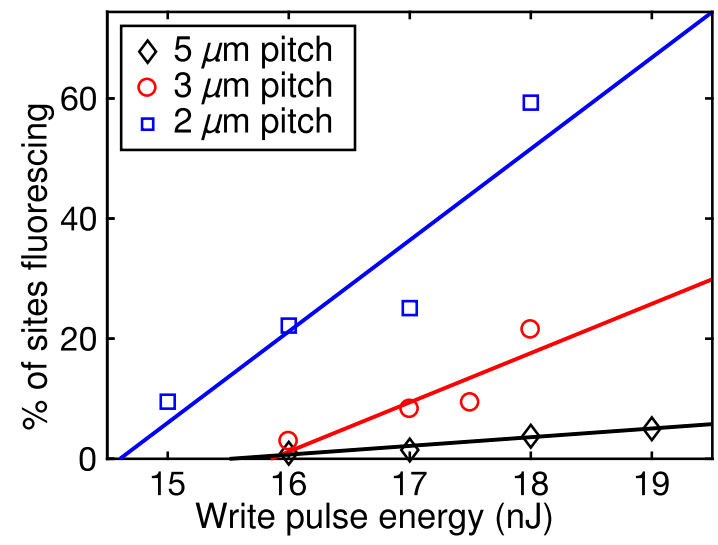

FIG. 6. Proportion of sites fluorescing after the annealing process.

\section{EXPERIMENTS}

We study this sample with our home-built scanning confocal microscope using ODMR with 532-nm excitation and single-photon counting modules for the broadband red fluorescence. The electron spins are coherently controlled with microwaves at 2.1-3 GHz. A 25-mT magnetic field is applied along the [111] direction for the spin coherence measurements, suppressing the periodic spin-echo collapse and revival [47]. Each spin coherence measurement takes 2-12 h, depending on the desired signal-to-noise ratio. The equipment is controlled using open-source QUDI software [48] with some of our modifications to enable automated data collection over many NVCs [49].

Figure 2 contains images from our scanning confocal microscope of one of the 3D arrays (array $\mathrm{M}$ ) before and after annealing. Before annealing, laser-written spots are visible due to the fluorescence of neutrally charged vacancies $V^{0}$, which are lattice sites in the diamond with missing carbon atoms. This is shown in the top row of Fig. 2. The characteristic $V^{0}$ fluorescence spectrum confirms the identity of these vacancies as shown in Fig. 5(a) of Appendix B. None of these preanneal spots were visible with our air objective, but these images were collected with our oil (a)

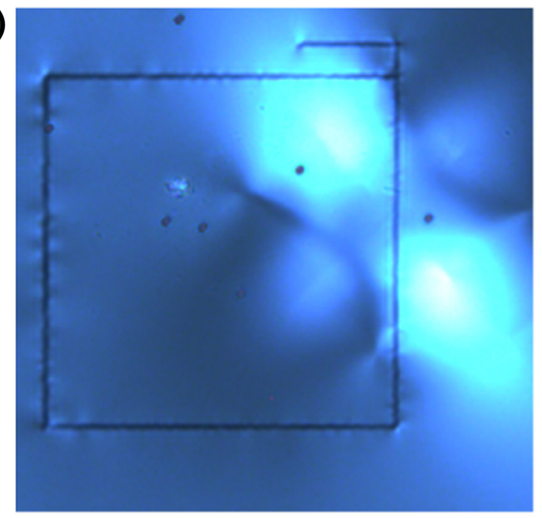

(c)

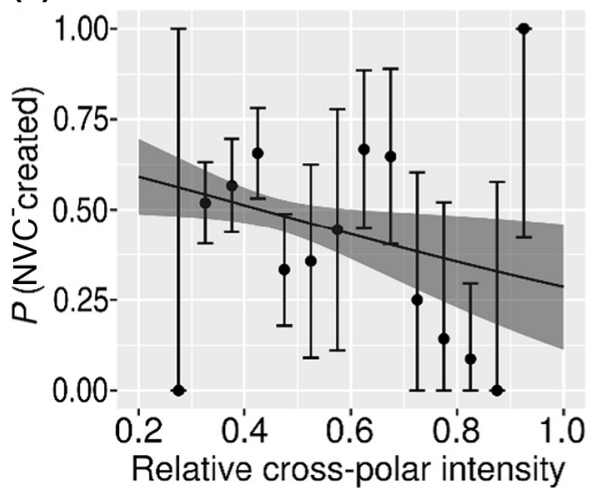

(b)

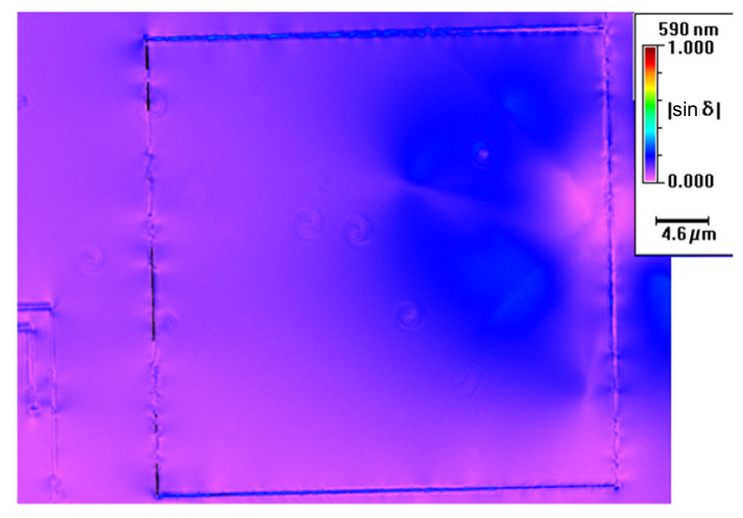

(d)

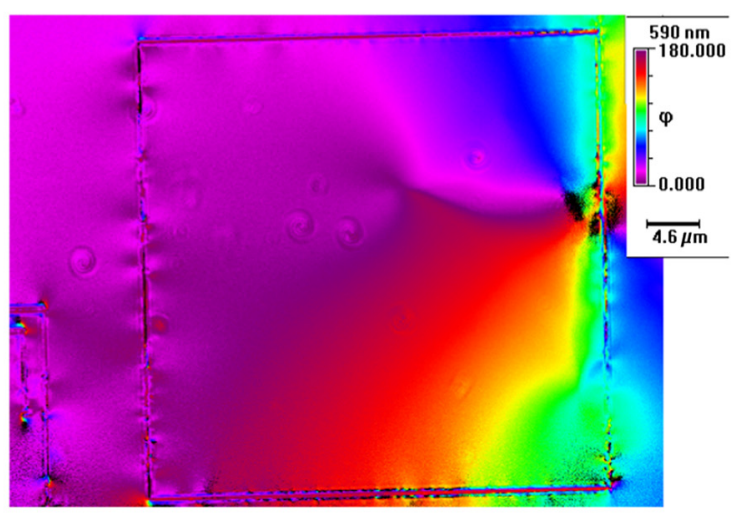

FIG. 7. Array I has the most variation in strain as determined by crossed-polarizer microscopy (a) and Metripol measurements of $|\sin (\delta)|$ (b) and orientation (d). These images are orientated to match confocal microscopy. (c) Probability of single or double NVC formation in any of the layers of array I, binned by relative crossed-polarizer intensity (normalized over the diamond). Metripol measurements show only one order of phase shift so the brightness in the cross-polar image indicates higher birefringence, and hence strain, aggregated through the diamond. The fit to a binary logistic regression model is shown with a 1 standard deviation confidence interval, however, the correlation is not significant $(p=0.3)$, indicating that NVC yield is not correlated with strain. The probability of forming NVCs is taken as the fraction of sites in each cross-polar brightness grouping which develop a single or double NVC (optically resolvable or not). 

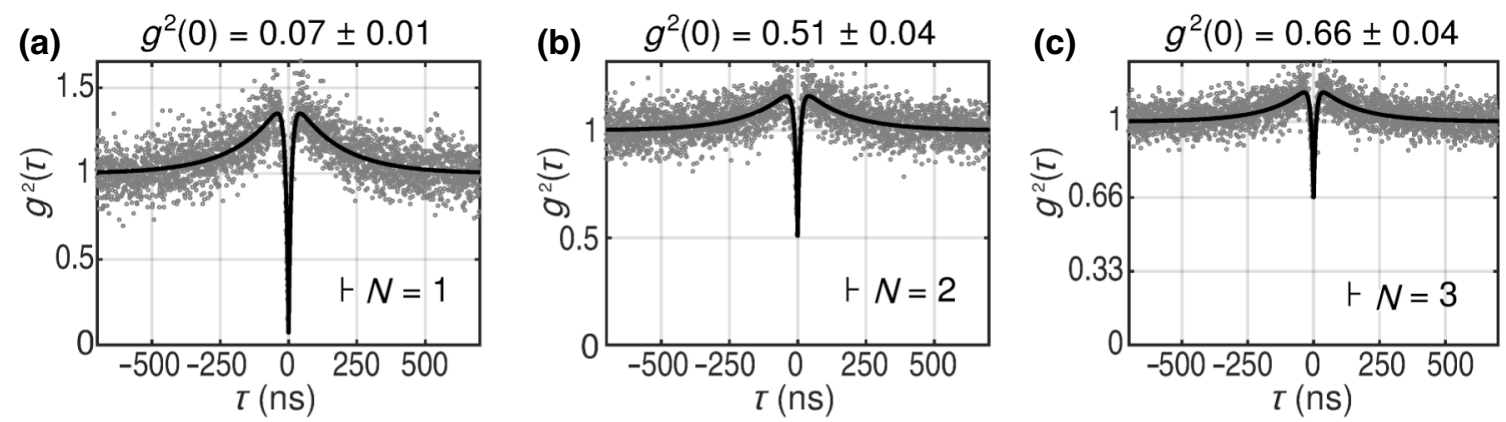

(d)

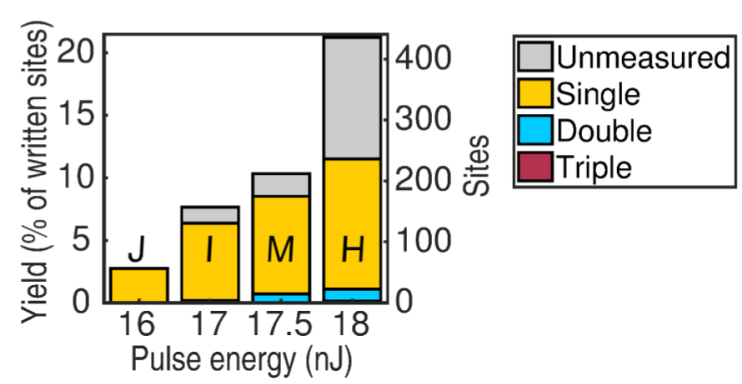

FIG. 8. Hanbury Brown-Twiss measurements are used to identify single NVC sites. (a)-(c) HBT photon arrival time auto-correlation measurements for three NVC sites in array H showing examples of single, double, and triple NVC classifications, respectively. (d) Statistics for all 3- $\mu \mathrm{m}$ pitch arrays, which show fluorescence, including incomplete measurement coverage of array $\mathrm{H}$ with approximately $20 \%$ yield of single NVCs, $2.5 \%$ double NVCs, and $0.3 \%$ triple NVCs, trading increased yield for a higher proportion of multiple NVC sites.

objective (the numerical aperture equals 1.4). We anneal the diamond at $1000{ }^{\circ} \mathrm{C}$ for $3 \mathrm{~h}$ in a nitrogen environment [25] and repeat the imaging, as shown in the bottom row of Fig. 2. The spots that can be seen are NVCs as confirmed by the characteristic fluorescence spectrum shown in Fig. 5(b) of Appendix B. We analyze the precision of the position for 167 single NVCs by three-dimensional fitting of the point spread function as shown in Appendix G. This reveals that the NVCs are in the desired locations to within $\pm 220 \mathrm{~nm}$ in the transverse $(X-Y)$ plane and $\pm 250 \mathrm{~nm}$ in the vertical $(Z)$ direction. The high precision in the $Z$ direction is due to the nonlinearity of the writing. The precision is probably limited by the concentration of nitrogen in this material, which is in the range from 1 to 5 parts per billion (ppb) corresponding to an average spacing between nitrogen atoms of 180 to $100 \mathrm{~nm}$. This implies that the vacancies generally bond to one of their nearest nitrogen dopants.

To check if the NVCs are single centers, we use automated Hanbury Brown-Twiss (HBT) experiments on over 600 sites to measure the photon arrival autocorrelation function $g^{2}(\tau)$, as shown in Fig. 3. We classify a site as a single emitter where $g^{2}(0)<0.5$, a double for $0.5 \leq g^{2}(0)<0.66$, and a triple for $0.66 \leq g^{2}(0)<0.75$. Lower boundary lines could have been chosen to account for the fact that NVCs can switch between different charge states and the different emission dipoles that result from different NVC orientations. For array M, $87 \%$ of the NVCs are single centers while $11 \%$ are doubles and $2 \%$ are triples. To reach higher yields, the sites with no NVCs should be repeatedly rewritten with another laser pulse and reannealed until no sites are empty. One of our previous papers reported single NVC creation with laser writing [25]: higher pulse energies were used, which led to a higher yield of single NVCs, but there were a similar number of the unwanted double and triple NVCs, which would lead to a lower yield of single NVCs in a repeat-until-success strategy. We greatly reduce this problem here. Appendix E contains confocal imaging and HBT statistics for some of the other arrays. Of the 2050 sites written in array M, 8\% develop into a single NVC, $0.6 \%$ produce a double NVC, and $<0.1 \%$ produce a triple NVC. This is consistent with Poissonian statistics $\left(0.0064=0.08^{2}\right.$ and $\left.0.0005=0.08^{3}\right)$, which may suggest that NVC creation is limited by the nitrogen density rather than by the highly nonlinear laser writing. We may expect that if there were an abundance of NVCs, the nonlinear nature of the laser writing would lead to more doubles and triples for a given yield of single NVCs.

Figure 4 shows measurements of the spin coherence from 22 of the single NVCs in the $3-\mu \mathrm{m}$ pitch arrays $\mathrm{M}$ and I, which are written with 17.5 and $17 \mathrm{~nJ}$ pulses, respectively. The longest room-temperature spin-echo coherence times without ${ }^{12} \mathrm{C}$ enrichment we find in the literature are $T_{2}=687 \mu \mathrm{s}$ [3] and $T_{2}=650 \mu \mathrm{s}$ [50], which are slightly below (but within the error of) our five longest times. The 

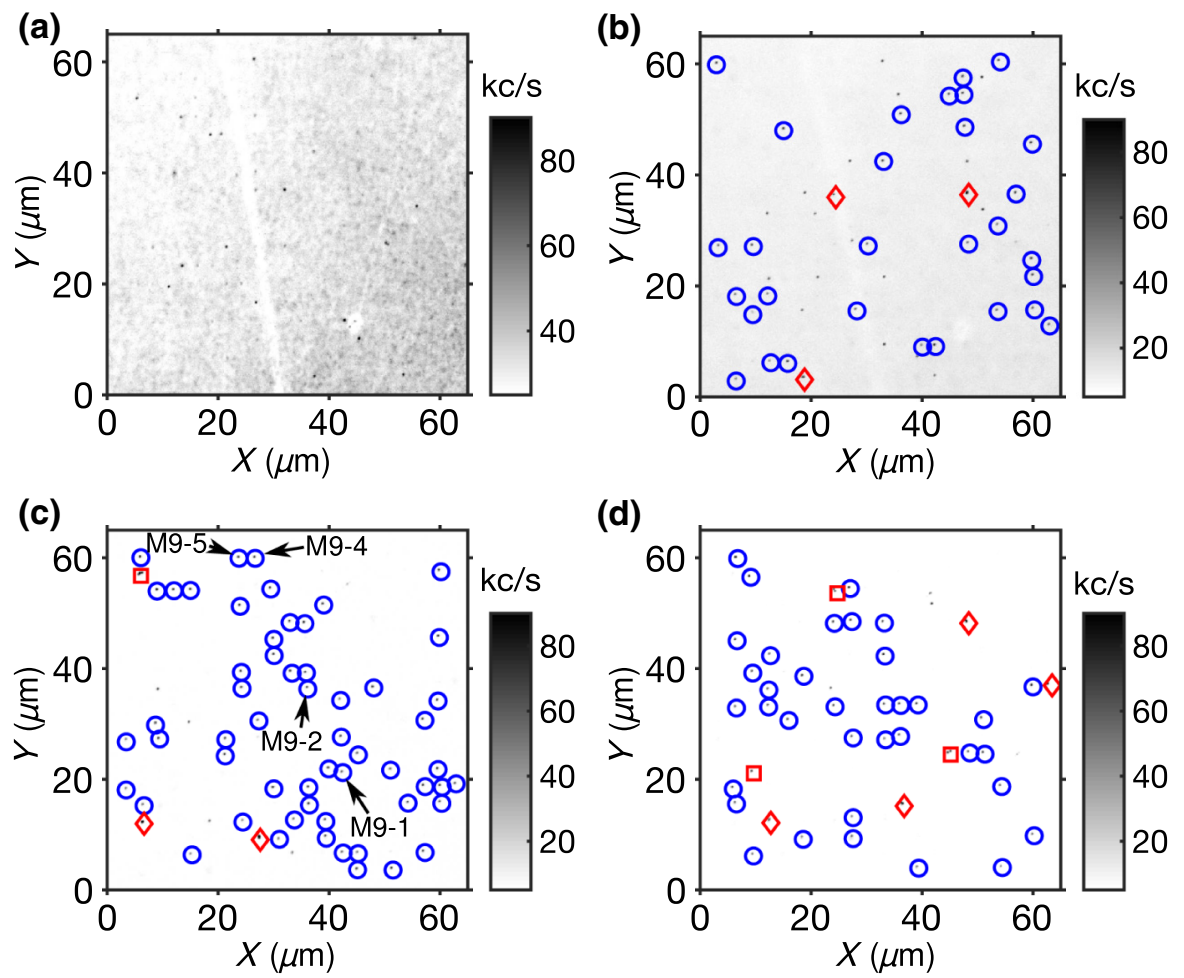

FIG. 9. Layers of array $M(17.5 \mathrm{~nJ}$, $3 \mu \mathrm{m}$ pitch) with single NVC (circle) and double NVC (diamond: determined by HBT, square: optically resolvable). Single NVC sites where $T_{2}$ is measured are labeled (M9-5 and M9-4 are the highlighted sites $\mathrm{NVC1}$ and $\mathrm{NVC2}$ in the main paper). (a)-(e) are, respectively, at depths of $3,6,9,12$, and $15 \mu \mathrm{m}$ below the surface.

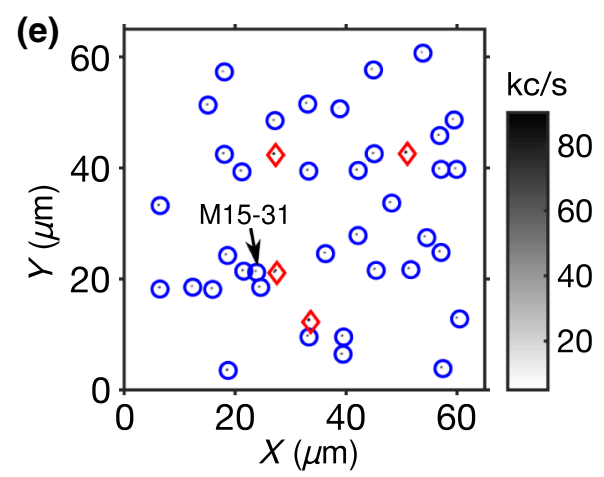

long times we measure demonstrate that our laser-writing technique does not introduce excess damage or impurities to the environment of the NVCs. Our new calibration step to ensure we use the optimum write-pulse energy may be needed to achieve this as that is a key difference from our previous work [25]. This allows the use of lower writepulse energies, which only create NVCs without leaving extra damage behind in the lattice.

Previous NVC optical entanglement work has applied electric fields to Stark shift the optical fluorescence frequency so that the two NVCs have indistinguishable emissions $[3,4]$. Figure 4(c) shows an NVC between two electrically conducting wires that we laser write in three dimensions at the same time as the arrays. It is known that these laser-written wires in diamond are graphitic and that they conduct with a de resistivity of around $0.1 \Omega \mathrm{cm}$ at room temperature [51].

\section{DISCUSSION AND CONCLUSION}

Further extensions of the work presented here could include studying the optical coherence [25] as a function of depth, and studying the spin coherence over a greater range of depths. Looking at shallower NVCs would reveal the effects of the surface and provide clearer statistics on the NVCs that are $6-\mu \mathrm{m}$ deep. The depth range presented here is ideal for solid immersion lenses, but approximately 2$\mu \mathrm{m}$ deep would be used for membrane experiments inside an optical cavity [23]. Testing the Stark shift that can be achieved at low temperatures with the graphitic wires is another important future experiment, as this would allow the optical emission of two NVCs to be brought to the same wavelength as required for optical entanglement [4]. To evaluate the usefulness of quantum sensing using gradiometry with two 2D arrays, it will be necessary to measure the crosstalk in the excitation and detection. Having 


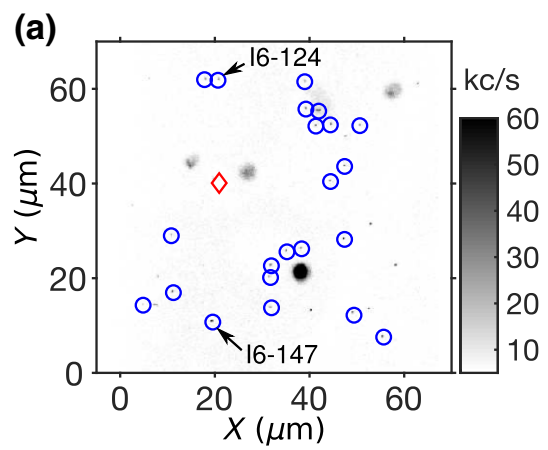

(c)

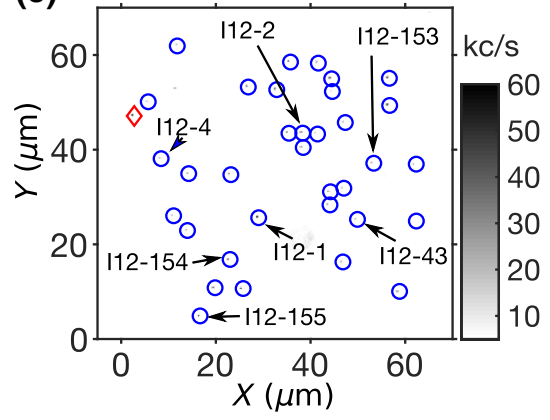

(b)

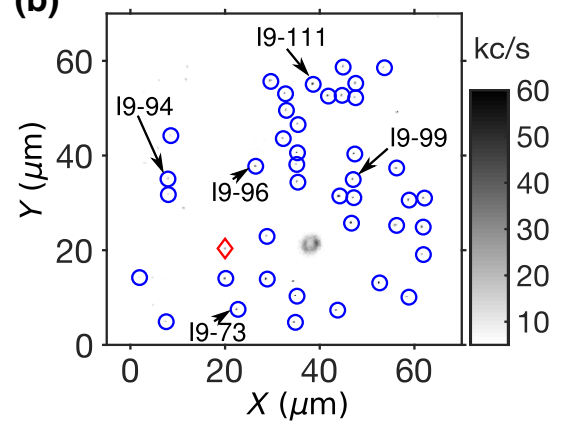

(d)

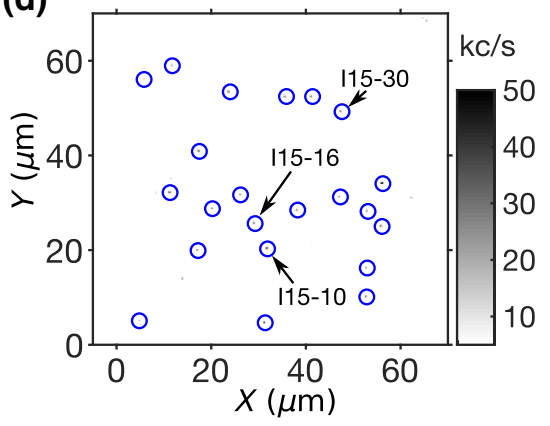

FIG. 10. Layers of array I (17 nJ, $3 \mu \mathrm{m}$ pitch) with single NVC (circle) and double NVC (diamond: determined by HBT, square: optically resolvable). Single NVC sites where $T_{2}$ is measured are labeled. (a)-(d) are, respectively, at depths of $6,9,12$, and $15 \mu \mathrm{m}$ below the surface. a larger spacing between the 2D arrays may help with this.

In conclusion, this work demonstrates creation of arrays of long-lived spin qubits in a solid-state system with alignment to control structures in three dimensions inside diamond, making this a compelling approach for engineering larger quantum systems. Finding that the spin coherence times at room temperature are as long as naturally occurring NVCs shows that laser writing is suitable for quantum computer designs calling for an array of many NVCs in one diamond. The data for this paper is available online [52].

\section{ACKNOWLEDGMENTS}

C.J.S.'s PhD is funded by the Royal Society and G.W.M. is supported by the Royal Society. P.H.'s PhD is cofunded by the Fraunhofer Centre for Applied Photonics. P.H. and P.L.D.'s PhDs are cofunded by the EPSRC Centre for Doctoral Training in Diamond Science and Technology (Grant No. EP/L015315/1). This work was supported by the EPSRC NQIT (Networked Quantum Information Technology) Hub (Grant No. EP/M013243/1) and by P.S.S.'s EPSRC fellowship (Grant No. EP/R004803/1).

\section{APPENDIX A: ARRAY LOCATIONS AND PARAMETERS}

Arrays A to $\mathrm{G}$ are fabricated as six layers at depths of $5,10,15,20,25$, and $30 \mu \mathrm{m}$. Each layer is written with 410 pulses in 20 rows and 21 columns of consistent pulse energy with a pitch of $5 \mu \mathrm{m}$. The write pulse energies of arrays A to $\mathrm{G}$ are, respectively: $19,18,17,16,15,14$, and $18 \mathrm{~nJ}$. Arrays $\mathrm{H}$ to $\mathrm{N}$ are fabricated as five layers at depths of 3, 6, 9, 12, and $15 \mu \mathrm{m}$. Each layer is written with 410 pulses in 20 rows and 21 columns of consistent pulse energy with a pitch of $3 \mu \mathrm{m}$. The write pulse energies of arrays $\mathrm{H}$ to $\mathrm{N}$ are, respectively: 18, 17, 16, 15, 14, 17.5 , and $18 \mathrm{~nJ}$. Arrays $\mathrm{O}$ to $\mathrm{V}$ are fabricated as four layers at depths of 4, 6, 8, and $10 \mu \mathrm{m}$. Each layer is written with 410 pulses in 20 rows and 21 columns of consistent pulse energy with a pitch of $2 \mu \mathrm{m}$. The write pulse energies of arrays $\mathrm{O}$ to $\mathrm{V}$ are, respectively: 18, 17, 16, 15, 14, $16,16.5$, and $17 \mathrm{~nJ}$. The labeling of the arrays is shown in Fig. 1(b).

\section{APPENDIX B: PHOTOLUMINESCENCE SPECTROSCOPY}

Photoluminescence (PL) spectra under excitation with a 532-nm laser are measured using an Andor Shamrock spectrograph with an iDus series silicon CCD through a multimode fiber.

Before the sample is annealed, PL spectra are collected such as that shown in Fig. 5(a), clearly showing the characteristic GR1 emission spectrum associated with neutral vacancies in diamond. No sites are visible by confocal microscopy with our oil objective in array $\mathrm{D}(16 \mathrm{~nJ})$ before annealing. Following the anneal, PL spectra are collected from sites including M9-4 (labeled NVC2 above), which clearly show the characteristic spectrum of $\mathrm{NVC}^{-}$ as in Fig. 5(b). The background emission from a nearby region of diamond is collected for an equal duration and 

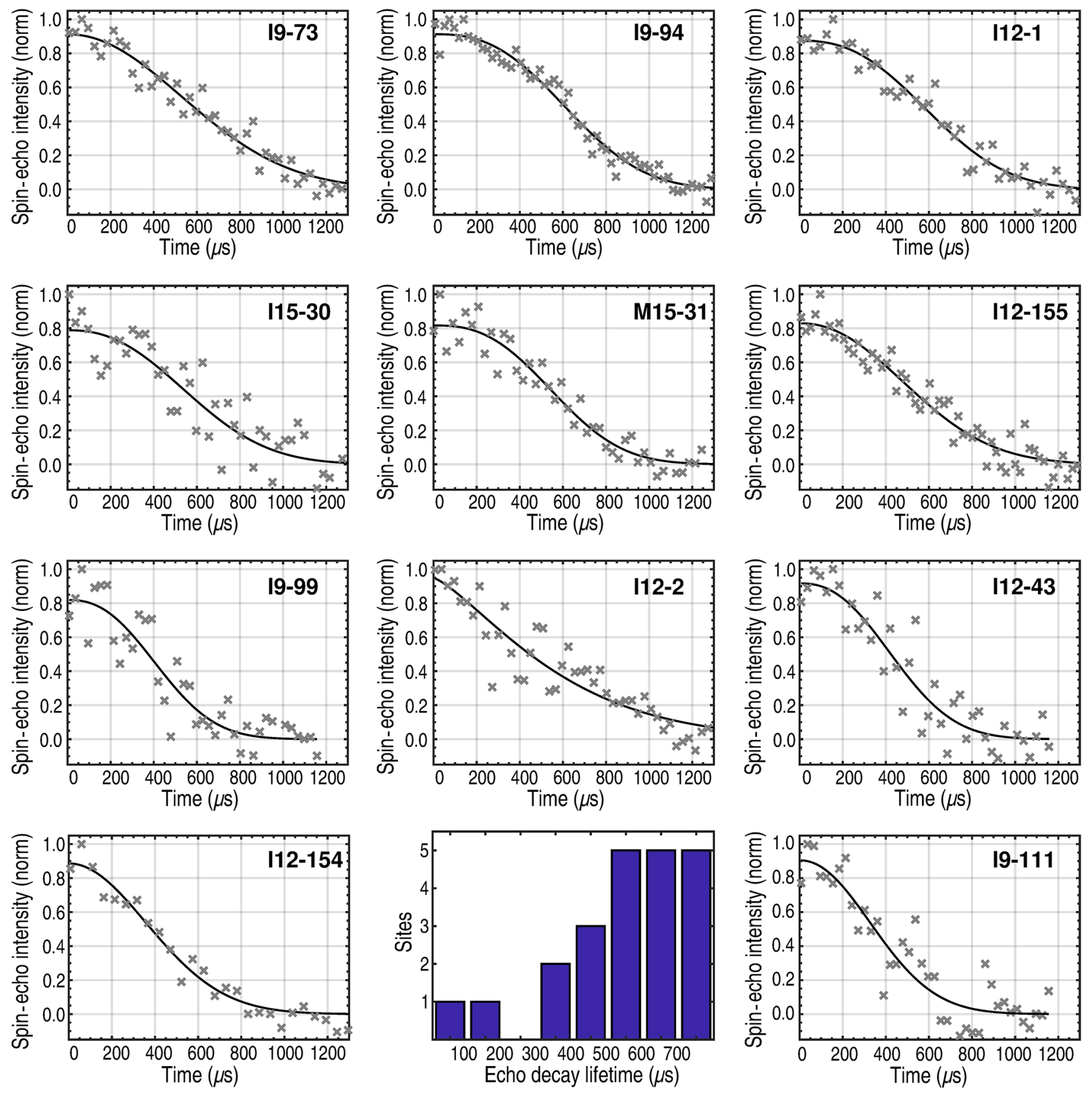

FIG. 11. Examples of $T_{2}$ spin-echo coherence time measurements from arrays $\mathrm{M}$ and I along with a histogram of the echo decay coherence times

subtracted to eliminate artifacts of spectrometer response, second-order Raman emission, and fluorescence from the immersion oil.

\section{APPENDIX C: NVC COLOR CENTER YIELD}

A narrow range of pulse energies are used to create three-dimensional arrays, each of which has a pitch of 2 , 3 , or $5 \mu \mathrm{m}$ in the $x, y$, and $z$ directions. The proportion of sites that developed NVCs are shown in Fig. 6. While the yield of NVCs in the 2- $\mu \mathrm{m}$ pitch arrays is high with a high proportion of single NVCs, the close packing of sites in depth make it more difficult to image individual sites in the $z$ direction. The 3- $\mu \mathrm{m}$ pitch array develops a good yield of well-spaced NVCs, which can be reliably characterized. Despite a higher pulse energy, the $5-\mu \mathrm{m}$ pitch array develops a lower yield of NVCs. This suggests that a smaller pitch leads to more damage, which is reasonable given that the sites are written on top of each other in the $z$ direction. 
TABLE I. Spin-echo coherence times $\left(T_{2}\right)$ for the sites measured and fitted to $A e^{-\left(t / T_{2}\right)^{n}}$.

\begin{tabular}{lllll}
\hline \hline Site & \multicolumn{2}{c}{ Coherence time $(\mu \mathrm{s})$} & \multicolumn{2}{c}{ Exponent } \\
\hline I9-73 & 730 & $(40)$ & 2.1 & $(0.4)$ \\
I9-94 & 730 & $(20)$ & 2.7 & $(0.3)$ \\
I9-96 & 710 & $(60)$ & 2.1 & $(0.6)$ \\
I12-1 & 710 & $(40)$ & 2.5 & $(0.5)$ \\
M9-5 (NVC1) & 706 & $(40)$ & 2.4 & $(0.4)$ \\
M9-4 (NVC2) & 690 & $(90)$ & 2.0 & $(0.8)$ \\
I15-30 & 680 & $(100)$ & 2.4 & $(1.1)$ \\
M15-31 & 650 & $(50)$ & 2.7 & $(0.7)$ \\
I12-155 & 640 & $(40)$ & 2.2 & $(0.4)$ \\
I12-2 & 630 & $(110)$ & 1.3 & $(0.4)$ \\
I15-10 & 570 & $(90)$ & 1.3 & $(0.4)$ \\
I12-43 & 520 & $(60)$ & 2.4 & $(1.0)$ \\
I6-147 & 520 & $(110)$ & 1.6 & $(0.8)$ \\
I12-154 & 510 & $(50)$ & 2.0 & $(0.5)$ \\
I9-99 & 500 & $(70)$ & 2.4 & $(1.2)$ \\
I9-111 & 448 & $(70)$ & 2.1 & $(0.9)$ \\
I12-4 & 430 & $(150)$ & 1.3 & $(0.7)$ \\
M9-2 & 420 & $(40)$ & 2.0 & $(0.5)$ \\
I12-153 & 390 & $(70)$ & 2.4 & $(1.4)$ \\
I15-16 & 360 & $(90)$ & 1.3 & $(0.6)$ \\
M9-1 & 200 & $(30)$ & 1.3 & $(0.4)$ \\
I6-124 & 25 & $(20)$ & 1.1 & $(1.2)$ \\
\hline \hline
\end{tabular}

\section{APPENDIX D: BIREFRINGENCE AND THE INFLUENCE OF STRAIN}

Array I has the region of highest birefringence, in the upper right quadrant of the images in Fig. 7, however throughout the arrays, $\Delta n<80 \times 10^{-6}$, which is comparable to low birefringence optical diamond and is relatively low for electronic grade diamond. The laser writing sites that do and do not develop fluorescing NVCs are not strongly correlated with the birefringence indicated by brightness through crossed polarizers in Fig. 7(c). The highest brightness corresponds to a birefringence averaged through the plate of approximately $\Delta n=80 \times 10^{-6}$.

\section{APPENDIX E: HANBURY BROWN-TWISS MEASUREMENTS}

Fluorescence from the NVCs being measured is split by a nonpolarizing beam splitter and the autocorrelation of the photon arrival events at two Excelitas near-infrared (NIR) enhanced single photon counting modules (SPCMs) is accumulated using a time tagger. The data are normalized by the accumulated autocorrelation for large $\tau$ and fitted using the photon arrival time autocorrelation model for a three-level system

$$
g^{(2)}(\tau)=1-d\left(c e^{-|\tau| / \tau_{2}}+(c-1) e^{-|\tau| / \tau_{3}}\right),
$$

with a modification to allow the depth $\left[d=1-g^{(2)}(0)\right]$ of the dip at $\tau=0$ to vary as a fit parameter. $\tau_{2}, \tau_{3}$, and $c$ relate to the lifetimes of states and the internal dynamics of the NVCs. The fitted $g^{(2)}(0)$ with measurement uncertainty inferred from the standard deviation of $g^{(2)}(\tau)$ at large $\tau$ is binned into $g^{(2)}(0)<0.5$ for single NVCs, $0.5 \leq g^{(2)}(0)<0.66$ for double NVCs, and $0.66 \leq g^{(2)}(0)<0.75$ for triple NVCs. Examples are shown (a)

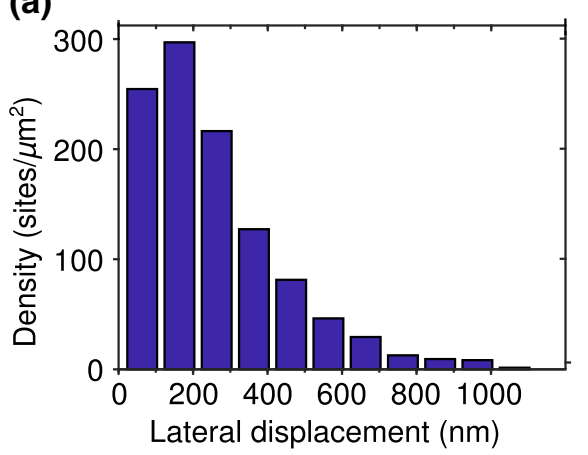

(c)

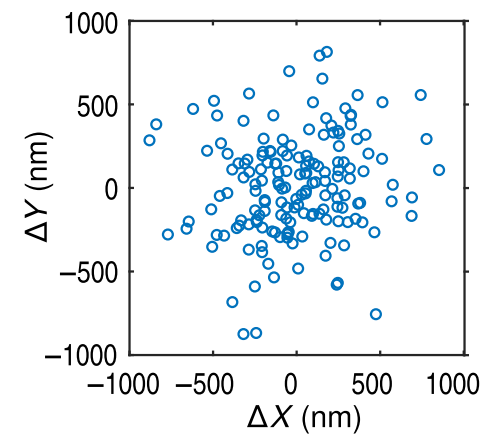

(b)

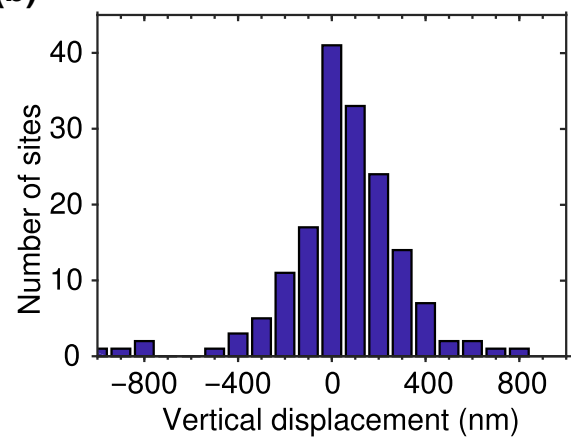

(d)

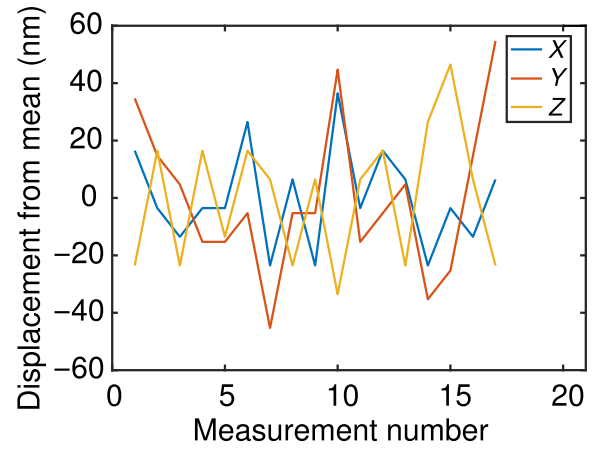

FIG. 12. Positioning precision of single nitrogen vacancy centers in array $\mathrm{M}$. (a),(c) In the $X-Y$ plane of the array. (b) In the vertical $Z$ direction. Standard deviations are 220 and $250 \mathrm{~nm}$, respectively, which is consistent with the mean separation of nitrogen in EL grade CVD diamond. (d) Change in the relative displacement between two points over time as measured by fitting the PSF and using a third point as a reference between each measurement. 
in Fig. 8. In cases where insufficient light is detected at automatically visited sites, some sites are inspected manually and where pairs of nearby NVCs confound the automated focusing, these are classified as optically resolved double sites as are sites where more than one optically distinct NVC can be seen in confocal imaging of the grid site. The full HBT statistics are shown in Fig. 8(d).

\section{APPENDIX F: SPIN COHERENCE MEASUREMENTS}

Arrays $\mathrm{M}$ and I are focused upon for measurements of the spin coherence: confocal images of their layers are shown in Figs. 9 and 10, respectively, which show both the distribution of single and double centers and the location of the NVCs where the spin-echo coherence time $\left(T_{2}\right)$ is measured. There is no obvious clustering of the multiple NVC sites or sites with long $T_{2}$ times. Some of the longest spin-echo coherence times measured as well as a histogram are shown in Fig. 11 and Table I gives all of the fitted coherence times.

\section{APPENDIX G: POSITION MEASUREMENTS}

In array $\mathrm{M}$, automated measurements of position are made relative to a reference single NVC using confocal microscopy using a PI Mars P-563 closed-loop nanopositioning stage. By fitting oversampled confocal scans of single NVCs with Gaussian profiles to approximate the point spread function (PSF) of the optical system, the positions of single centers relative to a repeatedly measured reference point are inferred to better than the diffraction limit in three dimensions. Repeated measurements show that this technique has an accuracy of around $20 \mathrm{~nm}$ over a 20-min period and remains self-consistent in the presence of slow drifts in sample position as shown in Fig. 12(d). The site positions are fitted to a grid of the design pitch and the position displacements reported are the measured displacements from the fitted orientation after rotation to compensate for sample tilt and skew as shown in Fig. 12.

[1] X. Rong, J. Geng, F. Shi, Y. Liu, K. Xu, W. Ma, F. Kong, Z. Jiang, Y. Wu, and J. Du, Experimental fault-tolerant universal quantum gates with solid-state spins under ambient conditions, Nat. Commun. 6, 8748 (2015).

[2] J. O'Gorman and E. T. Campbell, Quantum computation with realistic magic-state factories, Phys. Rev. A 95, 032338 (2017).

[3] H. Bernien, B. Hensen, W. Pfaff, G. Koolstra, M. S. Blok, L. Robledo, T. H. Taminiau, M. Markham, D. J. Twitchen, L. Childress, and R. Hanson, Heralded entanglement between solid-state qubits separated by three metres, Nature 497, 86 (2013).

[4] P. C. Humphreys, N. Kalb, J. P. J. Morits, R. N. Schouten, R. F. L. Vermeulen, D. J. Twitchen, M. Markham, and
R. Hanson, Deterministic delivery of remote entanglement on a quantum network, Nature 558, 268 (2018).

[5] A. Reiserer, N. Kalb, M. S. Blok, K. J. M. van Bemmelen, T. H. Taminiau, R. Hanson, D. J. Twitchen, and M. Markham, Robust Quantum-Network Memory Using Decoherence-Protected Subspaces of Nuclear Spins, Phys. Rev. X 6, 021040 (2016).

[6] M. H. Abobeih, J. Cramer, M. A. Bakker, N. Kalb, M. Markham, D. J. Twitchen, and T. H. Taminiau, Onesecond coherence for a single electron spin coupled to a multi-qubit nuclear-spin environment, Nat. Commun. 9, 2552 (2018).

[7] C. E. Bradley, J. Randall, M. H. Abobeih, R. C. Berrevoets, M. J. Degen, M. A. Bakker, M. Markham, D. J. Twitchen, and T. H. Taminiau, A 10-Qubit Solid-State Spin Register with Quantum Memory Up to One Minute, Phys. Rev. X 9, 031045 (2019).

[8] A. M. Stoneham, A. H. Harker, and G. W. Morley, Could one make a diamond-based quantum computer?, J. Phys. Condens. Matter 21, 364222 (2009).

[9] M. W. Doherty, C. A. Meriles, A. Alkauskas, H. Fedder, M. J. Sellars, and N. B. Manson, Towards a RoomTemperature Spin Quantum Bus in Diamond via Electron Photoionization, Transport, and Capture, Phys. Rev. X 6, 041035 (2016).

[10] N. Y. Yao, L. Jiang, A. V. Gorshkov, P. C. Maurer, G. Giedke, J. I. Cirac, and M. D. Lukin, Scalable architecture for a room temperature solid-state quantum information processor, Nat. Commun. 3, 800 (2012).

[11] J. O'Gorman, N. H. Nickerson, P. Ross, J. J. L. Morton, and S. C. Benjamin, A silicon-based surface code quantum computer, Npj Quantum Inform. 2, 15019 (2016).

[12] T. Gaebel, M. Domhan, I. Popa, C. Wittmann, P. Neumann, F. Jelezko, J. R. Rabeau, N. Stavrias, A. D. Greentree, S. Prawer, J. Meijer, J. Twamley, P. R. Hemmer, and J. Wrachtrup, Room-temperature coherent coupling of single spins in diamond, Nat. Phys. 2, 408 (2006).

[13] S. Yang, Y. Wang, D. D. B. Rao, T. Hien Tran, A. S. Momenzadeh, M. Markham, D. J. Twitchen, P. Wang, W. Yang, R. Stöhr, P. Neumann, H. Kosaka, and J. Wrachtrup, High-fidelity transfer and storage of photon states in a single nuclear spin, Nat. Photonics 10, 507 (2016).

[14] T. M. Babinec, B. J. M. Hausmann, M. Khan, Y. Zhang, J. R. Maze, P. R. Hemmer, and M. Lončar, A diamond nanowire single-photon source, Nat. Nanotechnol. 5, 195 (2010).

[15] C. Bonato, M. S. Blok, H. T. Dinani, D. W. Berry, M. L. Markham, D. J. Twitchen, and R. Hanson, Optimized quantum sensing with a single electron spin using real-time adaptive measurements, Nat. Nanotechnol. 11, 247 (2015).

[16] I. Gross, W. Akhtar, V. Garcia, L. J. Martínez, S. Chouaieb, K. Garcia, C. Carrétéro, A. Barthélémy, P. Appel, P. Maletinsky, J. V. Kim, J. Y. Chauleau, N. Jaouen, M. Viret, M. Bibes, S. Fusil, and V. Jacques, Real-space imaging of non-collinear antiferromagnetic order with a single-spin magnetometer, Nature 549, 252 (2017).

[17] P. Appel, E. Neu, M. Ganzhorn, A. Barfuss, M. Batzer, M. Gratz, A. Tschöpe, and P. Maletinsky, Fabrication of all diamond scanning probes for nanoscale magnetometry, Rev. Sci. Instrum. 87, 063703 (2016). 
[18] D. Le Sage, K. Arai, D. R. Glenn, S. J. DeVience, L. M. Pham, L. Rahn-Lee, M. D. Lukin, A. Yacoby, A. Komeili, and R. L. Walsworth, Optical magnetic imaging of living cells, Nature 496, 486 (2013).

[19] J. P. Hadden, J. P. Harrison, A. C. Stanley-Clarke, L. Marseglia, Y.-L. D. Ho, B. R. Patton, J. L. O’Brien, and J. G. Rarity, Strongly enhanced photon collection from diamond defect centers under microfabricated integrated solid immersion lenses, Appl. Phys. Lett. 97, 241901 (2010).

[20] A. Ródenas, M. Gu, G. Corrielli, P. Paiè, S. John, A. K. Kar, and R. Osellame, Three-dimensional femtosecond laser nanolithography of crystals, Nat. Photonics 13, 105 (2019).

[21] G. Wachter, S. Kuhn, S. Minniberger, C. Salter, P. Asenbaum, J. Millen, M. Schneider, J. Schalko, U. Schmid, A. Felgner, D. Hüser, M. Arndt, and M. Trupke, Silicon microcavity arrays with open access and a finesse of half a million, Light: Sci. Appl. 8, 37 (2019).

[22] D. Riedel, I. Söllner, B. J. Shields, S. Starosielec, P. Appel, E. Neu, P. Maletinsky, and R. J. Warburton, Deterministic Enhancement of Coherent Photon Generation from a Nitrogen-Vacancy Center in Ultrapure Diamond, Phys/ Rev. X 7, 031040 (2017).

[23] M. Ruf, M. Ijspeert, S. van Dam, N. de Jong, H. van den Berg, G. Evers, and R. Hanson, Optically coherent nitrogen-vacancy centers in micrometer-thin etched diamond membranes, Nano Lett. 19, 3987 (2019).

[24] C. A. McLellan, B. A. Myers, S. Kraemer, K. Ohno, D. D. Awschalom, and A. C. Bleszynski Jayich, Patterned formation of highly coherent nitrogen-vacancy centers using a focused electron irradiation technique, Nano Lett. 16, 2450 (2016).

[25] Y.-C. Chen, P. S. Salter, S. Knauer, L. Weng, A. C. Frangeskou, C. J. Stephen, S. N. Ishmael, P. R. Dolan, S. Johnson, B. L. Green, G. W. Morley, M. E. Newton, J. G. Rarity, M. J. Booth, and J. M. Smith, Laser writing of coherent colour centres in diamond, Nat. Photonics 11, 77 (2017).

[26] D. Scarabelli, M. Trusheim, O. Gaathon, D. Englund, and S. J. Wind, Nanoscale engineering of closely-spaced electronic spins in diamond, Nano Lett. 16, 4982 (2016).

[27] K. Ohno, F. J. Heremans, C. F. d. 1. Casas, B. A. Myers, B. J. Alemán, A. C. B. Jayich, and D. D. Awschalom, Three-dimensional localization of spins in diamond using 12C implantation, Appl. Phys. Lett. 105, 052406 (2014).

[28] J. Meijer, B. Burchard, M. Domhan, C. Wittmann, T. Gaebel, I. Popa, F. Jelezko, and J. Wrachtrup, Generation of single color centers by focused nitrogen implantation, Appl. Phys. Lett. 87, 261909 (2005).

[29] M. Lesik, P. Spinicelli, S. Pezzagna, P. Happel, V. Jacques, O. Salord, B. Rasser, A. Delobbe, P. Sudraud, A. Tallaire, J. Meijer, and J.-F. Roch, Maskless and targeted creation of arrays of colour centres in diamond using focused ion beam technology, Phys. Status Solidi (a) 210, 2055 (2013).

[30] C. D. Weis, A. Schuh, A. Batra, A. Persaud, I. W. Rangelow, J. Bokor, C. C. Lo, S. Cabrini, E. Sideras-Haddad, G. D. Fuchs, R. Hanson, D. D. Awschalom, and T. Schenkel, Single atom doping for quantum device development in diamond and silicon, Journal of Vacuum Science \& Technology B: Microelectronics and Nanometer Structures Processing, Measurement, and Phenomena 26, 2596 (2008).

[31] S. Pezzagna, D. Wildanger, P. Mazarov, A. D. Wieck, Y. Sarov, I. Rangelow, B. Naydenov, F. Jelezko, S. W. Hell, and J. Meijer, Nanoscale engineering and optical addressing of single spins in diamond, Small 6, 2117 (2010).

[32] P. Spinicelli, A. Dréau, L. Rondin, F. Silva, J. Achard, S. Xavier, S. Bansropun, T. Debuisschert, S. Pezzagna, J. Meijer, V. Jacques, and J. F. Roch, Engineered arrays of nitrogen-vacancy color centers in diamond based on implantation of $\mathrm{CN}$-molecules through nanoapertures, New J. Phys. 13, 025014 (2011).

[33] S. Sangtawesin, T. O. Brundage, Z. J. Atkins, and J. R. Petta, Highly tunable formation of nitrogen-vacancy centers via ion implantation, Appl. Phys. Lett. 105, 063107 (2014).

[34] S. Pezzagna, B. Naydenov, F. Jelezko, J. Wrachtrup, and J. Meijer, Creation efficiency of nitrogen-vacancy centres in diamond, New J. Phys. 12, 065017 (2010).

[35] S. Pezzagna, D. Rogalla, H. W. Becker, I. Jakobi, F. Dolde, B. Naydenov, J. Wrachtrup, F. Jelezko, C. Trautmann, and J. Meijer, Creation of colour centres in diamond by collimated ion-implantation through nano-channels in mica, Phys. Status Solidi (a) 208, 2017 (2011).

[36] M. H. Abobeih, J. Randall, C. E. Bradley, H. P. Bartling, M. A. Bakker, M. J. Degen, M. Markham, D. J. Twitchen, and T. H. Taminiau, Atomic-scale imaging of a 27nuclear-spin cluster using a single-spin quantum sensor, arXiv:1905.02095 (2019).

[37] S. B. van Dam, M. Walsh, M. J. Degen, E. Bersin, S. L. Mouradian, A. Galiullin, M. Ruf, M. Ijspeert, T. H. Taminiau, R. Hanson, and D. R. Englund, Optical coherence of diamond nitrogen-vacancy centers formed by ion implantation and annealing, Phys. Rev. B 99, 161203(R) (2019).

[38] B. Sotillo, V. Bharadwaj, J. P. Hadden, M. Sakakura, A. Chiappini, T. T. Fernandez, S. Longhi, O. Jedrkiewicz, Y. Shimotsuma, L. Criante, R. Osellame, G. Galzerano, M. Ferrari, K. Miura, R. Ramponi, P. E. Barclay, and S. M. Eaton, Diamond photonics platform enabled by femtosecond laser writing, Sci. Rep. 6, 35566 (2016).

[39] J. P. Hadden, V. Bharadwaj, B. Sotillo, S. Rampini, R. Osellame, J. D. Witmer, H. Jayakumar, T. T. Fernandez, A. Chiappini, C. Armellini, M. Ferrari, R. Ramponi, P. E. Barclay, and S. M. Eaton, Integrated waveguides and deterministically positioned nitrogen vacancy centers in diamond created by femtosecond laser writing, Opt. Lett. 43, 3586 (2018).

[40] V. V. Kononenko, I. I. Vlasov, V. M. Gololobov, T. V. Kononenko, T. A. Semenov, A. A. Khomich, V. A. Shershulin, V. S. Krivobok, and V. I. Konov, Nitrogenvacancy defects in diamond produced by femtosecond laser nanoablation technique, Appl. Phys. Lett. 111, 081101 (2017).

[41] Y.-C. Chen, B. Griffiths, L. Weng, S. S. Nicley, S. N. Ishmael, Y. Lekhai, S. Johnson, C. J. Stephen, B. L. Green, G. W. Morley, M. E. Newton, M. J. Booth, P. S. Salter, and J. M. Smith, Laser writing of individual nitrogen-vacancy defects in diamond with near-unity yield, Optica 6, 662 (2019). 
[42] S. Sangtawesin, B. L. Dwyer, S. Srinivasan, J. J. Allred, L. V. H. Rodgers, K. De Greve, A. Stacey, N. Dontschuk, K. M. O'Donnell, D. Hu, D. A. Evans, C. Jaye, D. A. Fischer, M. L. Markham, D. J. Twitchen, H. Park, M. D. Lukin, and N. P. de Leon, Origins of Diamond Surface Noise Probed by Correlating Single Spin Measurements with Surface Spectroscopy, Phys. Rev. X 9, 031052 (2019).

[43] C. L. Lee, E. Gu, M. D. Dawson, I. Friel, and G. A. Scarsbrook, Etching and micro-optics fabrication in diamond using chlorine-based inductivelycoupled plasma, Diam. Relat. Mater. 17, 1292 (2008).

[44] R. R. Gattass and E. Mazur, Femtosecond laser micromachining in transparent materials, Nat. Photonics 2, 219 (2008).

[45] R. D. Simmonds, P. S. Salter, A. Jesacher, and M. J. Booth, Three dimensional laser microfabrication in diamond using a dual adaptive optics system, Opt. Express 19, 24122 (2011).

[46] S. Lagomarsino, S. Sciortino, B. Obreshkov, T. Apostolova, C. Corsi, M. Bellini, E. Berdermann, and C. J. Schmidt, Photoionization of monocrystalline CVD diamond irradiated with ultrashort intense laser pulse, Phys. Rev. B 93, 085128 (2016).
[47] P. London, R. Fischer, I. Alvizu, J. R. Maze, and D. Gershoni, Local probing of nuclear bath polarization with a single electronic spin, Phys. Rev. B 92, 241117(R) (2015).

[48] J. M. Binder, A. Stark, N. Tomek, J. Scheuer, F. Frank, K. D. Jahnke, C. Müller, S. Schmitt, M. H. Metsch, T. Unden, T. Gehring, A. Huck, U. L. Andersen, L. J. Rogers, and F. Jelezko, Qudi: A modular python suite for experiment control and data processing, SoftwareX 6, 85 (2017).

[49] https://github.com/WarwickEPR/qudi (2019).

[50] N. Mizuochi, P. Neumann, F. Rempp, J. Beck, V. Jacques, P. Siyushev, K. Nakamura, D. J. Twitchen, H. Watanabe, S. Yamasaki, F. Jelezko, and J. Wrachtrup, Coherence of single spins coupled to a nuclear spin bath of varying density, Phys. Rev. B 80, 041201(R) (2009).

[51] B. Sun, P. S. Salter, and M. J. Booth, High conductivity micro-wires in diamond following arbitrary paths, Appl. Phys. Lett. 105, 231105 (2014).

[52] C. J. Stephen, B. L. Green, Y. N. D. Lekhai, L. Weng, P. Hill, S. Johnson, A. C. Frangeskou, P. L. Diggle, Y.-C. Chen, M. J. Strain et al., Data for: "Deep three-dimensional solid-state qubit arrays with long-lived spin coherence," https://wrap.warwick.ac.uk/128147 (2019). 